\title{
A novel method for assessing cerebral autoregulation in preterm infants using transfer function analysis
}

\author{
Zachary A. Vesoulis' ${ }^{1}$, Steve M. Liao', Shamik B. Trivedi' ${ }^{1}$ Nathalie El Ters' ${ }^{1}$ and Amit M. Mathur ${ }^{1}$
}

BACKGROUND: Autoregulatory dysfunction is an important contributor to brain injury in premature infants, particularly intraventricular hemorrhage (IVH). The autoregulatory system acts as a filter that dampens the systemic blood flow to follow a normal cerebral perfusion profile.

METHODS: Simultaneous arterial blood pressure and cerebral near-infrared spectroscopy (NIRS) data were collected from infants born before 28 wk estimated gestational age. The resulting data were preprocessed and then divided into nonoverlapping 20-min epochs. The transfer function estimate was calculated to determine dampening ability.

RESULTS: Sixty-two infants were prospectively recruited with a mean estimated gestational age of $25.4 \pm 1.3 \mathrm{wk}$ and birth weight of $832 \pm 199 \mathrm{~g} .67 \%$ were male, 24/62 had IVH, 17/62 received dopamine, 47/62 had antenatal steroid exposure, and 22/62 received fentanyl.

Advancing estimated gestational age and birth weight z-score predicted stronger dampening while African-American race and IVH of any grade predicted weaker dampening.

CONCLUSION: This preliminary report suggests an impairment in dampening ability associated with immaturity, decreased birth weight z-score, and African-American race. Decreased dampening is also associated with $\mathrm{IVH}$, although these results cannot distinguish between decreased dampening as an antecedent or sequela of IVH. These observations should be studied in a larger sample.

$\mathbf{T}$ he cerebral vascular autoregulatory system is a physiologic mechanism that maintains consistent and stable cerebral blood flow (CBF). It works, in part, by attenuating the effect of low-frequency oscillations in blood pressure by way of a highpass filter $(1,2)$. In the mid-19th century, Siegmund Mayer noted spontaneous rhythmic oscillations in blood pressure, with a period of approximately $10 \mathrm{~s}(0.1 \mathrm{~Hz})$. Later experimental evidence demonstrated that these oscillations, now called "Mayer waves," arise as the result of oscillations in the sympathetic vasomotor tone of arterial blood vessels throughout the body (3), coordinated via cytosolic signaling and leading to synchronized activation of smooth muscle cells (4).
Dysfunction of the cerebral autoregulatory system, and therefore loss of dampening of blood flow oscillations, leads to unstable CBF as demonstrated by Ocon et al. (5), who found strong transmission of $0.1 \mathrm{~Hz}$ oscillations in blood pressure to the cerebral vasculature (indicative of a loss in dampening) with an associated reduction in cerebral blood flow velocity in a series of patients with dysautonomia.

Recent approaches to quantifying cerebral autoregulation have focused on the relationship between the mean arterial blood pressure (MAP) and cerebral saturation measured by near-infrared spectroscopy (NIRS). Reliably testing the function of the cerebral autoregulatory system in the preterm population, however, has proven to be a difficult task, largely due to the broad diversity in neurophysiologic development, technical challenges associated with capturing data, the lack of a clear definition for hypotension, (6) and lack of a standardized analysis methodology. A variety of methods have been used including lowfrequency coherence $(7,8)$ and time-domain correlation $(9,10)$ with findings consistent with those of the older, more invasive approaches, i.e., that sick, preterm infants have more evidence of autoregulatory dysfunction $(11,12)$. While individually interesting, drawing larger conclusions from these studies has been very difficult due to a lack of a standardized approach to collecting data (e.g., sampling rates ranging $0.2-10 \mathrm{~Hz}$ ) and interpretation of results. Furthermore, these approaches fundamentally examine the statistical correlation between the MAP and the cerebral NIRS signal, inherently requiring an arbitrary threshold value and thereby converting a dynamic process to a binary one.

An alternate view of autoregulatory systems can be derived by repeatedly measuring how naturally occurring oscillations are transformed and reflected in the cerebral circulation. We hypothesize that impaired autoregulation will manifest as a loss of dampening, i.e., a greater amplitude of systemic blood pressure oscillations transmitted to the cerebral circulation. An approach to measure this dampening effect, termed transfer function analysis, has been used to evaluate cerebral autoregulation in healthy adults (13) and adults with autonomic failure (14). The strength of this approach is that it measures dampening on a continuum that may evolve across gestational age, medical illness, or time. 


\section{Articles | Vesoulisetal.}

In this study, we investigated the dampening ability of the cerebral autoregulatory system in preterm infants during the first $72 \mathrm{~h}$ of life using transfer function analysis. We hypothesized that (i) advancing gestational age would be associated with improved dampening ability, (ii) perinatal factors would modulate dampening ability, and (iii) intraventricular hemorrhage (IVH) would be associated with decreased dampening.

\section{RESULTS}

\section{Sample Characteristics and Data Quality}

Sixty-two infants were prospectively recruited for the study with a mean gestational age $25.4 \pm 1.3 \mathrm{wk}$, mean birth weight $833 \pm 199 \mathrm{~g}$, and 42/62 (67\%) were male. A comprehensive list of sample characteristics can be found in Table 1 .

The median postnatal age at recording start was $12 \mathrm{~h}$ (range: 5-24h) and a median of 204 twenty-minute data epochs (approximately $68 \mathrm{~h}$ ) were captured per infant. Preprocessing resulted in average rejection of $49 \%$ of collected data epochs leaving a median of 105 data epochs (range 48-348 data epochs) per infant in the final analysis. $61 \%$ of rejected data epochs occurred due to motion artifact, $35 \%$ of rejected epochs occurred due to desaturation, while the remaining $4 \%$ of epochs were rejected due to interruptions in the recording.

Table 1. Descriptive statistics of sample

\begin{tabular}{|c|c|}
\hline & Sample $(n=62)$ \\
\hline Gestational age at birth, mean $\pm S D$, weeks & $25.4 \pm 1.3$ \\
\hline Birth weight, mean $\pm S D$, grams & $833 \pm 199$ \\
\hline Male sex, $n(\%)$ & $42(67)$ \\
\hline \multicolumn{2}{|l|}{ Race } \\
\hline White, $n(\%)$ & $32(52)$ \\
\hline African-American, $n$ (\%) & $30(48)$ \\
\hline $\mathrm{SGA}, n(\%)$ & $5(8)$ \\
\hline \multicolumn{2}{|l|}{ Antenatal steroids } \\
\hline None, $n(\%)$ & $15(24)$ \\
\hline Partial course, $n(\%)$ & $19(31)$ \\
\hline Complete course, $n(\%)$ & $28(45)$ \\
\hline Chorioamnionitis ${ }^{\mathrm{b}}, n(\%)$ & $22(35)$ \\
\hline Antenatal magnesium sulfate exposure, $n(\%)$ & $28(45)$ \\
\hline Adequate prenatal care ${ }^{\mathrm{a}}, n(\%)$ & $46(74)$ \\
\hline Vaginal delivery, $n(\%)$ & $25(40)$ \\
\hline Delayed cord clamping, $n(\%)$ & $9(15)$ \\
\hline Mechanical ventilation at $72 \mathrm{~h}, n(\%)$ & $45(73)$ \\
\hline Dopamine exposure, $n(\%)$ & $17(27)$ \\
\hline Fentanyl exposure, $n(\%)$ & $22(35)$ \\
\hline \multicolumn{2}{|l|}{ Intraventricular hemorrhage } \\
\hline All grades, $n(\%)$ & $23(39)$ \\
\hline Grade III-IV, $n(\%)$ & $6(10)$ \\
\hline Died in the neonatal period, $n(\%)$ & $6(10)$ \\
\hline
\end{tabular}

In Figure 1, the dampening curve for an example infant is shown after ensemble averaging of 50, 150, and 300 data epochs respectively. The shape of the curve is consistent, with a "notch" at approximately $0.1 \mathrm{~Hz}$ (Mayer wave frequency) with an increasing degree of smoothness as more data epochs are incorporated in the ensemble average. The bottom panel of Figure 1 depicts the estimate of dampening at the Mayer wave frequency for the same infant as an increasing number of data epochs are averaged, demonstrating that the estimate stabilizes after averaging a minimum of 25 data epochs (approximately $8 \mathrm{~h}$ ), varying by no more than $0.5 \mathrm{~dB}$.

\section{The Dampening Curve and Gestational Age}

The mean dampening curve, grouped by gestational age, is shown in Figure 2 and demonstrates a remarkably similar shape, regardless of the gestational age of the infant, with a developmental trend noted across gestational age clusters with increasing degrees of dampening (more negative) with advancing maturity at birth.

\section{Multiple Linear Regression Model}

The multiple linear regression prediction model was statistically significant $(F(7,54)=9.26, P<0.01)$ and accounted for approximately $50 \%$ of the variance $\left(R^{2}=0.546\right.$, adjusted $\left.R^{2}=0.487\right)$. Advancing estimated gestational age at birth, chorioamnionitis, and birthweight $\mathrm{z}$-scores were independently associated with greater dampening. African-American race and diagnosis of IVH at any time during the first week of life (of any grade) were independently associated with less dampening. The complete model output is shown in Table 2.

\section{IVH}

In the overall group, 23/62 infants (37\%) had IVH of any grade while 6/23 (26\%) had severe (grade III or IV) IVH. Given the initial results demonstrating a link between decreased dampening and IVH, a second level of analysis was conducted to investigate the dampening ability of infants who had IVH during the first $3 \mathrm{~d}$ (and thus had contemporaneous measurement of dampening ability). We restricted the analysis of dampening to those infants $(15 / 23)$ where there was certainty about the IVH status during the monitoring period. For the remaining $8 / 23$ infants with IVH, the diagnosis was made outside of the monitoring window. In three of eight of these infants, there was no IVH noted on the initial ultrasound performed on the first day of life but follow-up imaging was not performed until $7 \mathrm{~d}$ of life or later. The other five infants did not have any imaging performed until $1 \mathrm{wk}$ of life. In either case, it is impossible to determine if the hemorrhage occurred during the data acquisition period, thus the reason for exclusion. A statistical comparison of these infant groups is provided in Table 3.

Frequency response curves were clustered by IVH status as shown in Figure 3. Visual inspection demonstrated a clear gap in the dampening ability between the groups across the entire frequency band, including the Mayer wave frequency. The binary logistic regression prediction model was statistically significant $\left(\chi^{2}=24.57, P<0.01\right)$ and accounted 

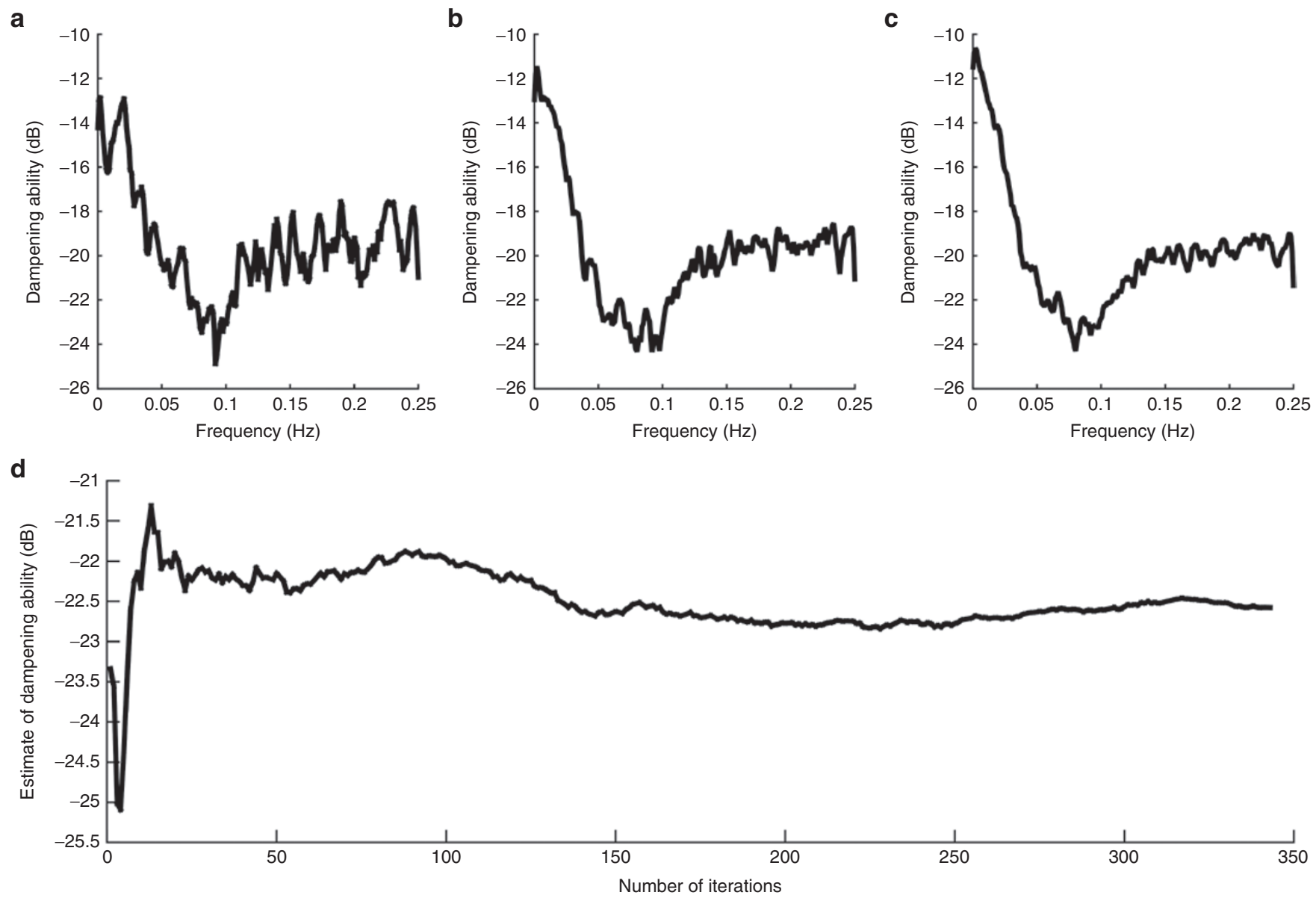

Figure 1. Data quality example. The dampening curve for a single subject is shown after ensemble averaging of (a) 50, (b) 150 , and (c) 300 data epochs respectively. The estimate of the dampening ability as the number of samples in the ensemble average is increased is shown in (d).

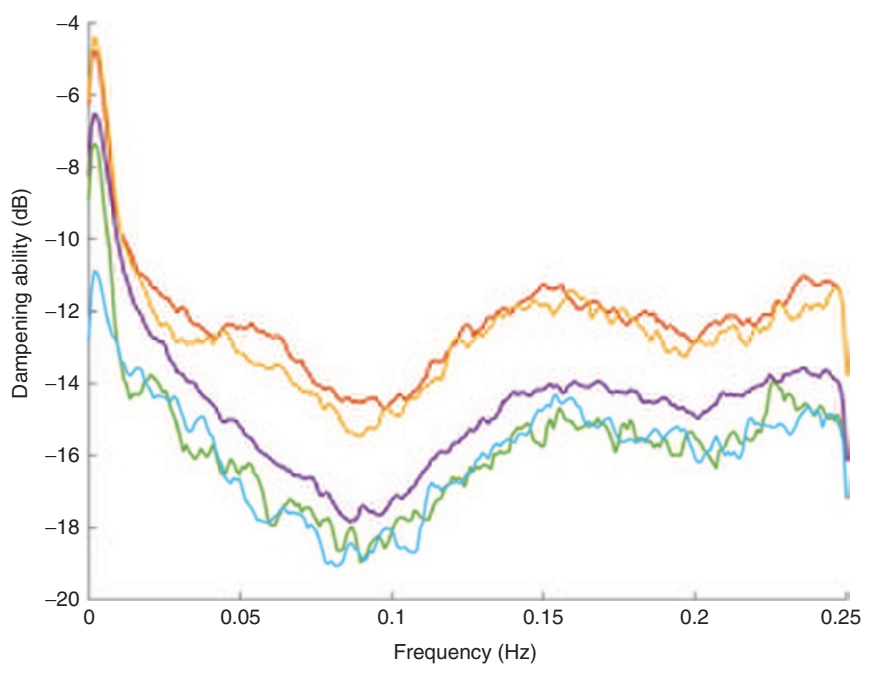

Figure 2. Dampening ability by gestational age. Broadband frequency response of the autoregulatory system by gestational age at birth. The response (dampening) of the autoregulatory system is indicated on the $y$-axis for discrete frequencies between 0 and $0.25 \mathrm{~Hz}$. Note the increased dampening at approximately $0.1 \mathrm{~Hz}$ (Mayer frequency band). Infants are grouped by gestational and color coded (red-24 wk $(n=17)$, yellow- $25 \mathrm{wk}(n=15)$, purple-26 wk $(n=19)$, green-27 wk $(n=5)$, blue-28 wk $(n=6))$. for approximately $30 \%$ of the variance $\left(R^{2}=0.385\right.$, adjusted $\left.R^{2}=0.275\right)$. Higher birth weight $\mathrm{z}$-score was independently associated with a lower likelihood of developing IVH, while decreased dampening and fentanyl exposure were associated with a greater likelihood of developing IVH. Complete model output is shown in Table 4. A separate analysis for infants with high-grade IVH was not possible due to the small number of infants in this category $(n=4)$.

\section{DISCUSSION}

In this study, we have demonstrated the feasibility of measuring cerebral autoregulation in preterm infants using NIRS and arterial blood pressure data collected continuously over the first $3 \mathrm{~d}$ of life. These data demonstrate evidence for dampening of spontaneous oscillations in systemic blood pressure by the cerebral autoregulatory system, particularly prominent at the Mayer wave frequency. Furthermore, this dampening ability increased as a function of advancing gestational age at birth and was decreased in infants who developed IVH. Our data build on findings of Rhee et al. (15), that there is ongoing development of the cerebral autoregulatory system during the second trimester. This is not surprising, given that simultaneous maturation of other organ systems occurs over the same 


\section{Articles $\mid$ vesoulisetal.}

Table 2. Multiple regression model for predictors of dampening in the Mayer wave frequency band

\begin{tabular}{|c|c|c|c|c|}
\hline \multirow[b]{2}{*}{ Factor } & \multicolumn{2}{|c|}{$\beta$ coefficient $^{a}$} & \multirow[b]{2}{*}{$P$ value } & \multirow[b]{2}{*}{ VIF } \\
\hline & Unstandardized & Standardized & & \\
\hline BW z-score & -0.04 & -0.26 & $0.01^{*}$ & 1.07 \\
\hline African-American race & 2.26 & 0.29 & $<0.01^{*}$ & 1.05 \\
\hline Prenatal care & -1.33 & -0.15 & 0.13 & 1.09 \\
\hline Chorioamnionitis $^{b}$ & -1.10 & -0.23 & $0.02^{*}$ & 1.10 \\
\hline
\end{tabular}

ancrease or decrease in magnitude of dampening from the mean (dB). ${ }^{b}$ Based on histologic examination of the placenta.

* denotes significance at $P \leq 0.05$. Model $R^{2}=0.546$, Adj. $R^{2}=0.487, P<0.01$.

BW, birth weight; VIF, variance inflation factor.

Table 3. Clinical characteristics by IVH status

\begin{tabular}{|c|c|c|c|}
\hline & $\begin{array}{c}\text { IVH } \\
(n=15)\end{array}$ & $\begin{array}{l}\text { No IVH } \\
(n=39)\end{array}$ & $\begin{array}{c}P \\
\text { value }\end{array}$ \\
\hline Gestational age, mean $\pm S D$, weeks ${ }^{a}$ & $25.1(1.4)$ & $25.6(1.4)$ & 0.288 \\
\hline Birth weight, mean $\pm S D$, grams ${ }^{a}$ & $876.7(228.5)$ & $823.2(197.5)$ & 0.475 \\
\hline Male sex, $n(\%)^{\mathrm{b}}$ & $11(73)$ & $25(64)$ & 0.748 \\
\hline African-American race, $n(\%)^{\mathrm{b}}$ & $8(53)$ & $18(46)$ & 0.764 \\
\hline $\mathrm{SGA}, n(\%)^{\mathrm{b}}$ & $1(7)$ & $4(10)$ & 1.00 \\
\hline Antenatal steroids, $n(\%)^{b}$ & $9(60)$ & $33(85)$ & 0.071 \\
\hline Antenatal MgSO4, $n(\%)^{\mathrm{b}}$ & $6(40)$ & $22(56)$ & 0.366 \\
\hline Chorioamnionitis $^{\mathrm{c}}, n(\%)^{\mathrm{b}}$ & $6(40)$ & $12(31)$ & 0.536 \\
\hline Adequate prenatal cared,$n(\%)$ & $10(67)$ & $30(77)$ & 0.498 \\
\hline Vaginal delivery, $n(\%)$ & $9(60)$ & $13(33)$ & 0.121 \\
\hline Delayed cord clamping, $n(\%)^{\mathrm{b}}$ & $3(20)$ & $4(10)$ & 0.382 \\
\hline Mech. ventilation at $72 \mathrm{~h}, n(\%)^{\mathrm{b}}$ & $13(87)$ & $24(62)$ & 0.105 \\
\hline Dopamine exposure, $n(\%)^{\mathrm{b}}$ & $5(33)$ & $10(26)$ & 0.735 \\
\hline Fentanyl exposure, $n(\%)^{b}$ & $8(53)$ & $10(25)$ & 0.105 \\
\hline Died in the neonatal period, $n(\%)^{b}$ & $0(0)$ & $4(10)$ & 0.567 \\
\hline
\end{tabular}

IVH, intraventricular hemorrhage; SGA, small for gestational age.

Comparison made using ${ }^{a}$ Mann-Whitney $U$-test or ${ }^{\circ}$ Fisher's Exact test (two-sided).

'Based on histologic examination of the placenta. ${ }^{\mathrm{d}}$ Defined as receiving at least $75 \%$ of

expected visits at time of birth $(27,28,32,33)$

period. The functional effect of the maturation is quite profound: the difference in dampening ability of the cerebral circulation between those born at 24 and $28 \mathrm{wk}(6 \mathrm{~dB})$ represents a $600 \%$ increase.

The mechanism underlying IVH is undoubtedly complex and multifactorial. Impaired autoregulation and resulting ischemia-reperfusion has been proposed as a contributing factor for the development of IVH in the past (16), a postulation which is somewhat borne out by our data. Nevertheless, the logistic regression model (of which dampening was a part) only explained about $30 \%$ of the variance predicting risk for IVH. Our findings are best interpreted in the context of vulnerability: impaired autoregulation does not equate to brain injury in every infant, but rather a diminishing ability to cope

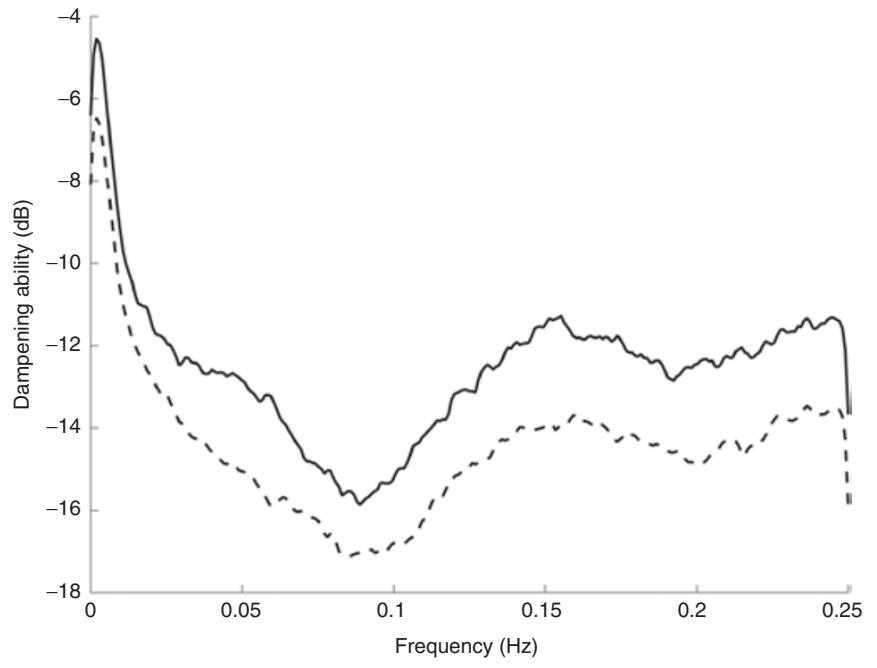

Figure 3. Magnitude of dampening vs. frequency for infants with intraventricular hemorrhage (IVH) (solid line) and without IVH (dashed line).

with perturbations in systemic perfusion that are common during this transitional period.

Our IVH data provide additional context for this vulnerability: those infants who develop IVH had a statistically worse ability to dampen systemic blood flow fluctuations, even when holding other clinical factors constant. A similar pattern of decreased dampening after brain injury has previously been described in adults with intracranial hemorrhage (17) or head trauma (18), with less dampening noted in those with more elevated intracranial pressures despite using a completely different technique for measurement of CBF (transcranial Doppler ultrasound). Caution should be urged in interpretation of these data. Due to the "snapshot" nature of cranial ultrasound (CUS) and the inability to precisely determine when IVH occurred, this methodology cannot distinguish between decreased dampening as an antecedent or sequela of IVH.

Given that the overarching objective was to develop a robust model of cerebral autoregulation, careful monitoring of data quality was of the utmost importance. The NICU is a noisy environment, and extracting a valid signal is challenging. We set strict criteria for data exclusion and did not interpolate 
Table 4. Binary logistic regression model for predictors of intraventricular hemorrhage, all grades

\begin{tabular}{|c|c|c|c|c|}
\hline \multirow[b]{2}{*}{ Factor } & \multicolumn{2}{|c|}{$\beta$ coefficient $^{\mathrm{a}}$} & \multirow[b]{2}{*}{$P$ value } & \multirow[b]{2}{*}{ VIF } \\
\hline & Unstandardized & Standardized & & \\
\hline BW z-score & -0.04 & 2.62 & $0.01 *$ & 1.50 \\
\hline Dampening & 0.34 & 2.96 & $<0.01^{*}$ & 2.07 \\
\hline $\begin{array}{l}\text { Estimated } \\
\text { gestational age }\end{array}$ & 0.38 & 1.16 & 0.37 & 1.81 \\
\hline Vaginal delivery & 1.39 & 1.53 & 0.11 & 1.22 \\
\hline Antenatal steroids & -1.84 & -1.71 & 0.07 & 1.22 \\
\hline Chorioamnionitis $^{b}$ & 1.27 & 1.34 & 0.16 & 1.30 \\
\hline Fentanyl exposure & 2.47 & 2.60 & $<0.01^{*}$ & 1.43 \\
\hline
\end{tabular}

missing data in order to reduce the likelihood of introducing error. The use of ensemble averaging allowed even further noise reduction, as graphically demonstrated in Figure 1. Despite the strict criteria, a clear view of autoregulatory system was indeed possible with as few as 25 data epochs (or about $8 \mathrm{~h}$ of data).

There is however, a potential bias introduced by rejection of such a significant quantity of data. This study methodology examines the relationship between blood pressure and cerebral oximetry in the frequency domain. Interpolation of a discontinuous time-series introduces error into the frequency domain representation of the data, becoming particularly prominent when the length of the discontinuity approaches the frequency of interest, in this case $0.1 \mathrm{~Hz}$ or $10 \mathrm{~s}$ (also known as the Gibbs phenomenon). Knowingly introducing error by interpolation was viewed to be an inferior choice in comparison to a, albeit unknown, possibility of bias due to exclusion. Future studies, with greater tolerance of noise or error may be able to derive the autoregulatory curve with even less data.

Although interpreted with great caution given the exploratory nature of the project, it is interesting to note the perinatal characteristics that were associated with improved dampening, namely chorioamnionitis and a higher birthweight $z$-score, perhaps as the result of more advanced physiologic maturation. Chorioamnionitis, in particular, has long been associated with rapid pulmonary maturation, the so-called "Watterberg effect" (19) and one could postulate that this systemic inflammation may induce extrapulmonary maturational changes. Similarly, higher birth weight $\mathrm{z}$-score is associated with a greater likelihood of survival, largely attributable to more mature organ functioning.

Given these findings, it was surprising to find no association between antenatal corticosteroids and improved cerebrovascular autoregulation, particularly given prior association with improved function of the sympathetic nervous system in preterm infants (20). However, there was significant heterogeneity in the number of steroid doses received prior to birth, with less than half of the infants receiving a full course prior to birth, potentially confounding this effect.
Although racial differences in autoregulatory function have not been previously described, a recent paper by Shankaran et al. (21) describes racially-based differences in the risk for IVH, with a reduced risk for IVH in Caucasian infants and no difference between male and female infants, possibly attributable to genetic factors as well as access prenatal care.

Future studies should evaluate the autoregulatory curve in a larger cohort, in particular one powered to assess the impact of antenatal steroids. A more rigorous CUS schedule will allow for a better estimate of IVH timing and allow for a more granular analysis of the dampening characteristics before, during and after hemorrhage.

A more complete understanding of the cerebral autroregulatory system will greatly benefit clinicians seeking to improve the care of preterm infants. Translation of this analysis method to real-time monitoring will enable clinicians to better implement neuroprotection strategies in this vulnerable population.

\section{METHODS}

\section{Participants}

Preterm infants, born prior to 28 completed weeks of gestation, were recruited as close to birth as possible for a prospective monitoring study during the first $72 \mathrm{~h}$ of life. The Washington University School of Medicine Human Research Protection Office approved the study and informed consent was obtained from the parents prior to data collection. Infants were only excluded if there was an antenatal diagnosis of a congenital malformation or if the clinical team did not elect to place an umbilical artery catheter. Historical data from our NICU indicated that more than $90 \%$ of infants meeting these criteria had an umbilical arterial line placed, making this an unlikely source of bias.

\section{Maternal and Infant Clinical Characteristics}

Perinatal factors were collected including prenatal visits and antenatal steroid or magnesium sulfate administration. Infant clinical characteristics were also collected from the infant's medical record including birth weight, birth weight $\mathrm{z}$-score (calculated from the Fenton growth charts (22)), sex, race, dopamine or fentanyl administration, and cranial ultrasound reports.

\section{Physiologic Data Collection}

NIRS. Cerebral tissue oxygen saturation $\left(\mathrm{SctO}_{2}\right)$ was obtained using 4-wavelength (690, 780, 805, and $850 \mathrm{~nm}$ ) near-infrared spectroscopy (Foresight, CAS Medical Systems, Branford, CT) with a transducer containing a fiber-optic emitter and one detector located $25 \mathrm{~mm}$ from the light source. The nonadhesive optode was placed on the frontoparietal scalp, and recording was conducted over the first $72 \mathrm{~h}$ after birth. As maintaining skin integrity in this vulnerable patient population was a high priority, a plan was developed prior to initiation of the study to limit the length of individual recordings to no more than $12 \mathrm{~h}$ (thus providing a "rest period") before repositioning the sensor approximately $1 \mathrm{~cm}$ laterally so as to prevent skin breakdown.

Regional cerebral tissue oxygen saturation measurements have previously been used to study autoregulation in preterm infants (23) and are less affected by motion artifact when compared to hemoglobin difference $(\mathrm{HbD})$, a different NIRS-derived oximetry measurement which measures the difference in concentrations between oxy- and deoxyhemoglobin (24)..

This study, as is the case for all cerebrovascular research using NIRS technology, is limited, to an extent, by the nature of the measurement, a regional mixed-venous saturation rather than a direct measurement of CBF itself. Nevertheless, there is agreement between NIRS and non-NIRS-derived measures of CBF, allowing NIRS to play an important role in continuous cerebral hemodynamic monitoring in patients who cannot be easily transported (i.e., very preterm infants). For example, Roche-Labarbe et al. (25) demonstrated a modest correlation $(r=0.22)$ between NIRS and transcranial Doppler ultrasound 
measures of CBF in very preterm infants, Kim et al. (26) noted a relatively strong correlation $(r=0.73)$ between NIRS and xenonenhanced CT imaging estimates of CBF in adult stroke patients, and Wintermark et al. (27) demonstrated an even stronger relationship $(r=0.88)$ between NIRS- and MRI-based estimates of CBF in term infants with severe encephalopathy.

MAP. The MAP was obtained from an umbilical arterial line (Argyle polyurethane umbilical vessel catheter, Covidien, Mansfield, MA) connected to a digital pressure transducer on the patient monitor (Intellivue MP70, Phillips Medical, Andover, MA).

Imaging. CUS examinations were performed in the first week of life, per the discretion of the clinical team. Intraventricular hemorrhage was graded on a scale from $0-4$ by radiologists blinded to the study data (28). Results of CUS exams performed outside of monitoring period were also captured to assess for later development of IVH.

Data collection. Both NIRS and MAP data streams were captured simultaneously on a laptop computer using custom data acquisition software (CAS Medical Systems) in a time-synchronized fashion at a sampling rate of $0.5 \mathrm{~Hz}$. Any interruptions in recording, such as "rest periods", withdrawal of blood through the catheter for laboratory testing, or infusion through the line, were noted by the bedside nurse or research assistant. The resulting data file was exported for offline analysis. Study data were collected and managed using REDCap electronic data capture tools (29) hosted at Washington University.

\section{Preprocessing}

The NIRS and MAP data streams were extracted from the source data file. Both data streams underwent multistep preprocessing to eliminate missing or invalid data. Twenty minutes (600 serial, nonoverlapping samples) of data were loaded at a time and inspected for (i) interrupted regions of the recording (as indicated in the research record), (ii) regions of the recording flagged by the NIRS device where it was not able properly measure the $\mathrm{SctO}_{2}$ (e.g., probe not in good contact with the skin), (iii) regions with sudden, nonphysiologic changes in the baseline or excessive variance, based on the slidingwindow motion artifact rejection algorithm proposed by Ayaz et al. (30), and (iv) regions of the trace where systemic saturation was below the lower limit of our target saturation guidelines $\left(\mathrm{SpO}_{2}<85 \%\right)$ given evidence that hypoxemia causes deviation from the expected arterial-venous contributions to the $\mathrm{SctO}_{2}$, making data during this time period unreliable (31). The entire data epoch was rejected if it failed at any one of these checks.

\section{Transfer Function Analysis}

The transfer function gain coefficient (dampening) was estimated for each remaining, error-free epoch using the following equation:

$$
H(f)=\frac{G_{i o}(f)}{G_{i i}(f)}
$$

Where $G_{\text {io }}(f)$ represents the cross-power spectrum between the input (MAP) and the output $\left(\mathrm{SctO}_{2}\right)$ and $G_{\mathrm{ii}}(f)$ represents the autopower spectrum of the input (MAP). Welch's modified periodogram method was used for spectral calculation. This calculation was made by first dividing each 20-min epoch, which is composed of 600 individual samples, into five segments overlapped by $75 \%$. The modified periodgram is computed for each segment and the results are averaged to provide a more accurate estimate of the spectral density. A Blackmann-Harris window was applied to the time-domain signal prior to spectral estimation to reduce spectral leakage.

The transfer function estimate was then calculated from 0.0 to 0.25 $\mathrm{Hz}$, limited by the Nyquist theorem given the sampling rate of 0.5 $\mathrm{Hz}$, yielding a frequency resolution of $0.0009 \mathrm{~Hz}$. Noise reduction was accomplished by ensemble averaging of the frequency response curve of each epoch within subjects and then between subjects, clustered by gestational age. The gain coefficient was logarithmically transformed to provide the amplitude of the dampening response measured in decibels $(\mathrm{dB})$ where $0 \mathrm{~dB}$ represents no transformation and $-10 \mathrm{~dB}$ represents a 10 -fold reduction in oscillation power. The mean gain coefficient in the Mayer wave frequency band (defined as $0.08-0.12$ $\mathrm{Hz}$ ) was calculated for each infant.

All signal processing was conducted using an in-house software package developed for MATLAB 8.4 (The MathWorks, Natick, MA).

\section{Statistical Analysis}

Covariate selection for the multiple linear regression model was undertaken by combining biologically plausible factors (gestational age, birth weight $\mathrm{z}$-score, antenatal steroid exposure) with important perinatal factors identified by forward stepwise multiple linear regression in order to develop a comprehensive, yet parsimonious model and avoid overfitting. Correlation between predictors was assessed using the variance inflation factor, a measure of the degree of multicollinearity where variance inflation factor $>5$ is indicative of highly correlated predictors.

A between-groups comparison was made for those with and without IVH by use of the Mann-Whitney $U$-test for continuous variables and a two-sided Fisher's Exact test for categorical variables. The influence of dampening on the outcome of IVH was assessed using binary logistic regression with covariate selection undertaken in the same fashion as in the previous step.

Statistical analysis including descriptive statistics and regression modeling were conducted using $\mathrm{R}$ version 3.1.0 (R Project for Statistical Computing, Vienna, Austria) and Minitab 17.0 (Minitab, State College, PA).

\section{ACKNOWLEDGMENTS}

The authors wish to thank Ben Palanca, MD for his assistance in optimizing the error correction algorithms, Anthony Barton for his tireless efforts in data collection, and CAS Medical Systems for their generous equipment loan.

\section{STATEMENT OF FINANCIAL SUPPORT}

This work was supported by the following grants: (i) Intellectual and Developmental Disabilities Research Center (IDDRC) at Washington University (NIH/NICHD P30 HD062171); (ii) Washington University Institute of Clinical and Translational Sciences KL2 Training Program (NIH/NCATS KL2 TR000450); (iii) The Barnes-Jewish Hospital Foundation and the Washington University Institute of Clinical and Translational Sciences Clinical and Translational Funding Program (NIH/NCATS UL1 TR000448).

Disclosure: The authors have no financial ties or potential/perceived conflicts of interest with any of the products or manufacturers described in this manuscript.

\section{REFERENCES}

1. Kuo TB, Chern CM, Sheng WY, Wong WJ, Hu HH. Frequency domain analysis of cerebral blood flow velocity and its correlation with arterial blood pressure. J Cereb Blood Flow Metab 1998;18:311-8.

2. Diehl RR, Linden D, Lücke D, Berlit P. Phase relationship between cerebral blood flow velocity and blood pressure. A clinical test of autoregulation. Stroke 1995;26:1801-4.

3. Julien C. The enigma of Mayer waves: Facts and models. Cardiovasc Res 2006;70:12-21.

4. Aalkjaer C, Nilsson H. Vasomotion: cellular background for the oscillator and for the synchronization of smooth muscle cells. Br J Pharmacol 2005; 144:605-16.

5. Ocon AJ, Medow MS, Taneja I, Clarke D, Stewart JM. Decreased upright cerebral blood flow and cerebral autoregulation in normocapnic postural tachycardia syndrome. Am J Physiol Heart Circ Physiol 2009;297: H664-73.

6. Bowen JR, Paradisis M, Shah D. Decreased aEEG continuity and baseline variability in the first 48 hours of life associated with poor short-term outcome in neonates born before 29 weeks gestation. Pediatr Res 2010;67: 538-44.

7. Soul JS, Hammer PE, Tsuji M, et al. Fluctuating pressure-passivity is common in the cerebral circulation of sick premature infants. Pediatr Res 2007;61:467-73.

8. Wong FY, Leung TS, Austin T, et al. Impaired autoregulation in preterm infants identified by using spatially resolved spectroscopy. Pediatrics 2008;121:e604-11. 
9. Chock VY, Ramamoorthy C, Van Meurs KP. Cerebral autoregulation in neonates with a hemodynamically significant patent ductus arteriosus. J Pediatr 2012;160:936-42.

10. Alderliesten T, Lemmers PM, Smarius JJ, van de Vosse RE, Baerts W, van Bel F. Cerebral oxygenation, extraction, and autoregulation in very preterm infants who develop peri-intraventricular hemorrhage. J Pediatr 2013;162:698-704.e2.

11. Lou HC, Lassen NA, Friis-Hansen B. Impaired autoregulation of cerebral blood flow in the distressed newborn infant. J Pediatr 1979;94:118-21.

12. Ment LR, Duncan CC, Ehrenkranz RA, et al. Intraventricular hemorrhage in the preterm neonate: timing and cerebral blood flow changes. J Pediatr 1984;104:419-25.

13. Zhang R, Zuckerman JH, Giller CA, Levine BD. Transfer function analysis of dynamic cerebral autoregulation in humans. Am J Physiol 1998;274(1 Pt 2):H233-41.

14. Blaber AP, Bondar RL, Stein F, et al. Transfer function analysis of cerebral autoregulation dynamics in autonomic failure patients. Stroke 1997;28:1686-92.

15. Rhee CJ, Fraser CD 3rd, Kibler K, et al. The ontogeny of cerebrovascular pressure autoregulation in premature infants. J Perinatol 2014;34:926-31.

16. Volpe JJ. Intraventricular hemorrhage in the premature infant-current concepts. Part I. Ann Neurol 1989;25:3-11.

17. Nakagawa K, Serrador JM, LaRose SL, Sorond FA. Dynamic cerebral autoregulation after intracerebral hemorrhage: A case-control study. BMC Neurol 2011;11:108.

18. Howells $\mathrm{T}$, Elf $\mathrm{K}$, Jones PA, et al. Pressure reactivity as a guide in the treatment of cerebral perfusion pressure in patients with brain trauma. J Neurosurg 2005;102:311-7.

19. Watterberg KL, Demers LM, Scott SM, Murphy S. Chorioamnionitis and early lung inflammation in infants in whom bronchopulmonary dysplasia develops. Pediatrics 1996;97:210-5.

20. Romejko-Wolniewicz E, Teliga-Czajkowska J, Czajkowski K. Antenatal steroids: can we optimize the dose? Curr Opin Obstet Gynecol 2014;26:77-82.

21. Shankaran S, Lin A, Maller-Kesselman J, et al.; Gene Targets for Intraventricular Hemorrhage Study. Maternal race, demography, and health care disparities impact risk for intraventricular hemorrhage in preterm neonates. J Pediatr 2014;164:1005-1011.e3.

22. Fenton TR, Kim JH. A systematic review and meta-analysis to revise the Fenton growth chart for preterm infants. BMC Pediatr 2013;13:59.
23. Caicedo A, De Smet D, Naulaers G, et al. Cerebral tissue oxygenation and regional oxygen saturation can be used to study cerebral autoregulation in prematurely born infants. Pediatr Res 2011;69:548-53.

24. van Bel F, Lemmers P, Naulaers G. Monitoring neonatal regional cerebral oxygen saturation in clinical practice: value and pitfalls. Neonatology 2008;94:237-44.

25. Roche-Labarbe N, Carp SA, Surova A, et al. Noninvasive optical measures of $\mathrm{CBV}, \mathrm{StO}(2), \mathrm{CBF}$ index, and $\mathrm{rCMRO}(2)$ in human premature neonates' brains in the first six weeks of life. Hum Brain Mapp 2010;31: 341-52.

26. Kim MN, Durduran T, Frangos S, et al. Noninvasive measurement of cerebral blood flow and blood oxygenation using near-infrared and diffuse correlation spectroscopies in critically brain-injured adults. Neurocrit Care 2010;12:173-80.

27. Wintermark P, Hansen A, Warfield SK, Dukhovny D, Soul JS. Near-infrared spectroscopy versus magnetic resonance imaging to study brain perfusion in newborns with hypoxic-ischemic encephalopathy treated with hypothermia. Neuroimage 2014;85 Pt 1:287-93.

28. Papile LA, Burstein J, Burstein R, Koffler H. Incidence and evolution of subependymal and intraventricular hemorrhage: a study of infants with birth weights less than 1,500 gm. J Pediatr 1978;92:529-34.

29. Harris PA, Taylor R, Thielke R, Payne J, Gonzalez N, Conde JG. Research electronic data capture (REDCap)-a metadata-driven methodology and workflow process for providing translational research informatics support. J Biomed Inform 2009;42:377-81.

30. Ayaz H, Izzetoglu M, Shewokis PA, Onaral B. Sliding-window motion artifact rejection for Functional Near-Infrared Spectroscopy. Conf Proc IEEE Eng Med Biol Soc 2010;2010:6567-70.

31. Wong FY, Alexiou T, Samarasinghe T, Brodecky V, Walker AM. Cerebral arterial and venous contributions to tissue oxygenation index measured using spatially resolved spectroscopy in newborn lambs. Anesthesiology 2010;113:1385-91.

32. Debiec KE, Paul KJ, Mitchell CM, Hitti JE. Inadequate prenatal care and risk of preterm delivery among adolescents: a retrospective study over 10 years. Am J Obstet Gynecol 2010;203:122.e1-6.

33. Kotelchuck M. An evaluation of the Kessner Adequacy of Prenatal Care Index and a proposed Adequacy of Prenatal Care Utilization Index. Am J Public Health 1994;84:1414-20. 